\title{
Mean-Variance Portfolio Selection with Margin Requirements
}

\author{
Yuan Zhou ${ }^{1,2}$ and Zhe $W u^{1}$ \\ ${ }^{1}$ School of Mathematics, Fudan University, Shanghai 200433, China \\ ${ }^{2}$ Department of Mathematics and Statistics, University of South Florida, Tampa, FL 33620-5700, USA
}

Correspondence should be addressed to Yuan Zhou; zhouy@mail.usf.edu

Received 18 November 2012; Revised 23 February 2013; Accepted 24 February 2013

Academic Editor: Cedric Yiu

Copyright (C) 2013 Y. Zhou and Z. Wu. This is an open access article distributed under the Creative Commons Attribution License, which permits unrestricted use, distribution, and reproduction in any medium, provided the original work is properly cited.

\begin{abstract}
We study the continuous-time mean-variance portfolio selection problem in the situation when investors must pay margin for short selling. The problem is essentially a nonlinear stochastic optimal control problem because the coefficients of positive and negative parts of control variables are different. We can not apply the results of stochastic linearquadratic (LQ) problem. Also the solution of corresponding Hamilton-Jacobi-Bellman (HJB) equation is not smooth. Li et al. (2002) studied the case when short selling is prohibited; therefore they only need to consider the positive part of control variables, whereas we need to handle both the positive part and the negative part of control variables. The main difficulty is that the positive part and the negative part are not independent. The previous results are not directly applicable. By decomposing the problem into several subproblems we figure out the solutions of HJB equation in two disjoint regions and then prove it is the viscosity solution of HJB equation. Finally we formulate solution of optimal portfolio and the efficient frontier. We also present two examples showing how different margin rates affect the optimal solutions and the efficient frontier.
\end{abstract}

\section{Introduction}

Modern portfolio theory was introduced by Markowitz in $1952[1,2]$. It is a theory of finance which attempts to minimize risk for a given level of expected return, by carefully choosing the proportions of various assets. In the theory variance of portfolio return was chosen as measure of the risk by Markowitz. Markowitz also established concept of the efficient frontier of the optimal portfolio: it is a curve showing the relation of the best possible expected level of return with respect to its level of risk (the standard deviation of the portfolio's return). This theory has been widely accepted both in financial industry and academy. There are lots of extensions and applications in the discrete time models [3]. Relatively there are less discussions of the mean-variance portfolio problem about continuous-time model [4-6]. In 2000, Zhou and $\mathrm{Li}$ [5] solved the continuous time mean-variance problem without short selling constraint by using the stochastic linear quadratic optimal control theory. Li et al. [6] in 2002 considered mean-variance portfolio selection with short selling prohibiting condition by solving HJB equation.

In financial market, the potential loss on a short sale can be huge when the price of the security goes up, therefore in practice the short seller will be required to post margin or collateral to cover possible losses. In this paper, we consider the situation that short selling is allowed and deposit of certain percentage margin according to the shorting is needed to avoid loss by default of short seller. We also want to investigate how margin rate $\lambda$ affect the optimal portfolio. In the extreme cases, when $\lambda=0$, it is the case short selling is free of margin and in another case $\lambda=\infty$ means that short selling is not allowed.

There is some literature [7-9] that discussed the margin requirements for portfolio selections. For example, Cuoco and Liu [7] examined the optimal consumption and investment choices and the cost of hedging contingent claims in the presence of margin requirements. They established existence of optimal policies by using martingale and duality techniques [10] under general assumptions on the securities price process and the investors preferences. However, the conditions for utility functions in [7] fail for mean-variance problem.

We use the model from [7], formulate a closed form of the optimal policies. The main difficulty lies when we use the approach of [6]: the positive part and the negative part of control variables $\pi$ have different coefficients; if we define new 
control variables $\left(\pi^{+}, \pi^{-}\right)$then the new volatility matrix is singular and moreover $\pi^{+}, \pi^{-}$are not independent therefore the key lemma for optimization is not applied in our case. We carefully decompose the problem into several subproblems and figure out the solutions of HJB equation in two regions and then prove it is the viscosity solution of HJB equation. Finally we formulate solution of optimal portfolio and the efficient frontier.

\section{Problem Formulation}

First, we prescribe a few notations. Let $(\Omega, \mathscr{F}, P)$ be a complete probability space and $\{W(t), 0 \leq t \leq T\}$ a standard $d$-dimensional Brownian motion defined on the probability space $\left\{\mathscr{F}_{t}^{W}\right\}_{t \geq 0}$ the natural filtration generated by $W(\cdot) . \mathcal{N}$ denotes the set composed of all the $P$ null set and its subsets; define the augmented filtration as $\mathscr{F}_{t}:=\sigma\left(\mathscr{F}_{t}^{W} \cup \mathcal{N}\right)$, for all $t \in[0, T]$, where $\mathscr{F}_{t}^{W}=\sigma\left(W_{s} \mid s \in[0, t]\right)$, then $\left\{\mathscr{F}_{t}\right\}_{t \geq 0}$ is complete and continuous filtration.

We define $a^{+}:=\max \{0, a\}, a^{-}:=\max \{0,-a\}$ for the positive part and negative part of a real number $a . \mathbf{R}^{n}$ represents $n$-dimensional real Euclidean space. $\mathbf{R}_{+}^{n}:=\{x \in$ $\left.\mathbf{R}^{n}, x_{i} \geq 0, i=1, \ldots, n\right\}$ is nonnegative subset of $n$-dimensional real Euclidean space. $L_{\mathscr{F}}^{2}\left(0, T ; \mathbf{R}^{n}\right):=\{f(\cdot) \mid f$ : $[0, T] \times \Omega \rightarrow \mathbf{R}^{n}, f(t)$ is $\mathscr{F}_{t}$ measurable for all $t \in[0, T]$, and $\left.E \int_{0}^{T}\|f(t)\|^{2} d t<+\infty\right\}$.

We consider the Merton's market model. There are $d+1$ securities, trading continuously in finite time horizon $[0, T]$. Assuming the interest rate is $r>0$, the time $t$ price of the risk-free asset satisfies

$$
d P_{0}(t)=r(t) P_{0}(t) d t, \quad t \in[0, T]
$$

with the initial price $P_{0}(0)=p_{0}>0$. The remaining $d$ risky assets prices at time $t$ are, respectively, $P_{1}(t), \ldots, P_{d}(t)$, which satisfy the following stochastic differential equations:

$$
\begin{gathered}
d P_{i}(t)=P_{i}(t)\left[b_{i}(t) d t+\sum_{j=1}^{d} \sigma_{i j}(t) d W_{j}(t)\right], \quad t \in[0, T], \\
P_{i}(0)=p_{i}>0,
\end{gathered}
$$

where $b(t)=\left(b_{1}(t), \ldots, b_{d}(t)\right)^{T}$ is the appreciation rate of the risky assets and $\sigma(t)=\left(\sigma_{i j}(t)\right)_{d \times d}$ is the volatility matrix.

Throughout the paper, we assume the following.

(A1) Function $r(t), b(t), \sigma(t)$ are deterministic and piecewise continuous on $[0, T]$.

(A2) Non-degenerate Condition: there exists a constant $\delta>0$, s.t.

$$
\sigma(t) \sigma(t)^{T} \geq \delta I, \quad \forall t \in[0, T]
$$

We also define the relative risk coefficient

$$
\theta(t):=\sigma(t)^{-1}(t)[b(t)-r(t) \mathbf{1}], \quad \forall t \in[0, T],
$$

where $\mathbf{1}=(1, \ldots, 1)^{T}$.
Let $v>0$ be initial wealth of the investor. Suppose $\pi_{i}(t), i=0,1, \ldots, d$ is time $t$ total wealth of the investor on $i$ th asset, $\pi(t):=\left(\pi_{1}(t), \ldots, \pi_{n}(t)\right)^{T}$ is time $t$ wealth vector of risky assets. Then time $t$ total wealth of the investor is $X(t)=\sum_{i=0}^{d} \pi_{i}(t)=\pi_{0}(t)+\mathbf{1}^{T} \pi(t)$. Suppose the investor is self-financing, then

$$
\begin{gathered}
d X(t)=\left\{r(t) X(t)+[b(t)-r(t) \mathbf{1}]^{T} \pi(t)\right. \\
\left.-\lambda r(t) \mathbf{1}^{T} \pi^{-}(t)\right\} d t+\pi^{T}(t) \sigma(t) d W(t), \\
t \in[0, T], \\
X(0)=v,
\end{gathered}
$$

where $\lambda \geq 0$ is the margin rate.

Note. When $\lambda=0$, there is no margin requirement for short selling and it has been discussed in [5].

In general, the terminal wealth $X(T)$ of the investor is a random variable. In the mean-variance problem, people consider $\mu=E[X(T)]$ as his return whereas variance of $X(T)$ as his risk. Therefore mean-variance problem is to minimize the risk of the investment for fixed expected return $\mu=$ $E[X(T)]$ :

$$
\operatorname{var}[X(T)]=E[X(T)-E[X(T)]]^{2}=E[X(T)-\mu]^{2} .
$$

The portfolio selection problem can be formulated as

$$
\begin{array}{ll}
\min & J(\mu, \pi(\cdot))=\operatorname{var}[X(T)] \\
\text { s.t. } & \left\{\begin{array}{l}
E[X(T)]=\mu, \\
\pi(\cdot) \in L_{\mathscr{F}}^{2}\left(0, T, \mathbf{R}^{d}\right), \\
X(\cdot) \text { satisfies }(5),
\end{array}\right.
\end{array}
$$

where the expected return is chosen as $\mu \geq \mu_{0}:=v e^{\int_{0}^{T} r(s) d s}$. If the investor puts all the money in the risk-free asset, his return will be $\mu_{0}$ with zero risk. Since the investor will always choose high return with low risk, therefore the lowest expected return must be $\mu_{0}$.

For fixed $\mu \geq \mu_{0}$, the optimal solution $\pi^{*}(\mu)$ is called efficient strategy. Corresponding minimal variance is $V(\mu)=$ $\min J(\mu, \pi(\cdot))$ and $(V(\mu), \mu)$ is called efficient point. When $\mu$ runs all over $\left[\mu_{0}, \infty\right)$, the set constructed by all the efficient points in variance-mean plane is called the efficient frontier.

\section{A Class of Stochastic Control Problem}

The problem mentioned in the previous section is a special class of stochastic nonlinear quadratic control problem. As the state equation is nonlinear on control variables $\pi$, the results of LQ problem can not apply. In this part, we will solve this control problem. 
Considering one dimensional system

$$
\begin{gathered}
d x(t)=\left[A(t) x(t)+B_{1}(t) \pi(t)-B_{0}(t) \pi^{-}(t)+f(t)\right] d t \\
+\pi^{T}(t) D_{1}^{T}(t) d W(t), \\
x(s)=y \in \mathbf{R},
\end{gathered}
$$

where $0 \leq s \leq T, A(t) \in \mathbf{R}_{+}, B_{0}^{T}(t), B_{1}^{T}(t) \in \mathbf{R}_{+}^{d}, D_{1}(t) \in \mathbf{R}^{d \times d}$, we assume matrix $D_{1}(t)$ is non-degenerate. These coefficients are all deterministic and piecewise continuous on $[0, T]$, therefore they are all bounded on $[0, T]$.

The class of admissible controls is the set $u_{s}=L_{\mathscr{F}}^{2}(s, T$, $\mathbf{R}^{d}$ ). For any given $\pi(\cdot) \in u_{s}$, by the result of stochastic differential equations theory there is a unique solution $x(\cdot ; y$, $\pi(\cdot)) \in L_{\mathscr{F}}^{2}(s, T, \mathbf{R})$ of $(8)$, and $(x(\cdot), \pi(\cdot))$ is called feasible pair. Our goal is to find admissible $\pi(\cdot) \in u_{s}$ to minimize the following cost function

$$
J(s, y ; \pi(\cdot))=\frac{1}{2} E[x(T)]^{2} .
$$

We define the value function of (9)

$$
V(s, y)=\inf _{\pi(\cdot) \in u_{s}} J(s, y ; \pi(\cdot)) .
$$

Obviously, it is nonnegative.

3.1. HJB Equation. We will try to find the optimal value function $V(s, y)$. According to stochastic optimal control theory [11], the value function is satisfying the following HJB equation:

$$
\begin{gathered}
v_{t}(t, x)+\inf _{u \in \mathbf{R}^{d}}\left\{v_{x}(t, x) g(t, x, u)+\frac{1}{2} v_{x x}(t, x)\right. \\
\left.\times u^{T} D_{1}^{T}(t) D_{1}(t) u\right\}=0, \\
v(T, x)=\frac{1}{2} x^{2},
\end{gathered}
$$

where $g(t, x, u)=A(t) x(t)+B_{1}(t) u-B_{0}(t) u^{-}+f(t)$. Our idea is to find local solution firstly, then prove that it is the viscosity solution of HJB equation and finally construct the optimal control by using verification theorem.

In order to find solution of the HJB equation, it is clear that for fixed $(t, x) \in[0, T] \times \mathbf{R}^{d}$ if $v_{x x}(t, x)>0$ (that will be proved later) we must solve

$$
\min _{u \in \mathbf{R}^{d}}\left\{\frac{1}{2} u^{T} D_{1}^{T}(t) D_{1}(t) u+\alpha(t, x)\left(B_{1}(t) u^{+}-B_{2}(t) u^{-}\right)\right\},
$$

where $B_{2}(t)=B_{0}(t)+B_{1}(t), \alpha(t, x)=v_{x}(t, x) / v_{x x}(t, x)$. We need the following lemma [10].

Lemma 1. If $D \in \mathbf{R}^{d \times d}$ and $|D| \neq 0$ then there is a unique $\widehat{z} \in$ $\mathbf{R}_{+}^{d}$, s.t.

$$
\left\|\left(D^{T}\right)^{-1} b+\left(D^{T}\right)^{-1} \widehat{z}\right\|^{2}=\min _{z \in \mathbf{R}_{+}^{d}}\left\|\left(D^{T}\right)^{-1} b+\left(D^{T}\right)^{-1} z\right\|^{2} .
$$

For nonnegative constant $\alpha$, the unique minimizer of

$$
h(z):=\frac{1}{2} z^{T} D^{T} D z-\alpha z^{T} b
$$

over $z \in \mathbf{R}_{+}^{d}$ is $\alpha \hat{\lambda}, \hat{\lambda}=D^{-1}\left(D^{T}\right)^{-1} \widehat{b}$, here $\widehat{b}=b+\widehat{z}$. Furthermore, $\widehat{z}^{T} \hat{\lambda}=0$ and

$$
h(\alpha \widehat{\lambda})=-\frac{1}{2} \alpha^{2}\left\|\left(D^{T}\right)^{-1} \hat{b}\right\|^{2}
$$

To solve the HJB equation (11), above lemma is not directly applicable. Now we introduce some transformation.

Note for any $u \in \mathbf{R}^{d}$, there is (may be not unique) a matrix $C=\operatorname{diag}\left(c_{1}, \ldots, c_{d}\right)$ with $c_{i}=1$ or -1 for $i=1, \ldots, d$, such that $u=C w, w \in \mathbf{R}_{+}^{d}, u^{+}=C^{+} w, u^{-}=C^{-} w$. Then

$$
\begin{aligned}
& \frac{1}{2} u^{T} D_{1}^{T} D_{1} u+\alpha\left(B_{1} u^{+}-B_{2} u^{-}\right) \\
& \quad=\frac{1}{2} w^{T} C^{T} D_{1}^{T} D_{1} C w+\alpha\left(B_{1} C^{+}-B_{2} C^{-}\right) w
\end{aligned}
$$

Define space $\mathscr{D}=\left\{C=\operatorname{diag}\left(c_{1}, \ldots, c_{d}\right)\right.$ with $c_{i}=1$ or -1 for $i=1, \ldots, d\}$.

We have following important lemma.

Lemma 2. If $\alpha$ is negative, there is at least one $b_{1} \in \mathbf{R}^{d}$ and $a$ minimizer $\alpha \widehat{\lambda}_{1}$ of

$$
h(u):=\frac{1}{2} u^{T} D_{1}^{T} D_{1} u+\alpha\left(B_{1} u^{+}-B_{2} u^{-}\right)
$$

over $\mathbf{R}^{d}$ and $h\left(\alpha \widehat{\lambda}_{1}\right)=-(1 / 2) \alpha^{2}\left\|b_{1}\right\|^{2}$ for all $\alpha<0$.

If $\alpha$ is positive, there is at least one $b_{2} \in \mathbf{R}^{d}$ and a minimizer $\alpha \widehat{\lambda}_{2}$ of $h(u)$ over $\mathbf{R}^{d}$ and $h\left(\alpha \widehat{\lambda}_{2}\right)=-(1 / 2) \alpha^{2}\left\|b_{2}\right\|^{2}$ for all $\alpha>0$.

Proof. In order to solve (12), we fixed a diagonal matrix $C$ with diagonals either $c_{i}=1$ or -1 for $i=1,2, \ldots, d$. Let

$$
h_{C}(w):=\frac{1}{2} w^{T} C^{T} D_{1}^{T} D_{1} C w+\alpha\left(B_{1} C^{+}-B_{2} C^{-}\right) w .
$$

If $\alpha<0$, take $D=D_{1} C$ and $b=\left(B_{1} C^{+}-B_{2} C^{-}\right)^{T}$. Since $D$ is nonsingular, by Lemma 1 , there exist vectors $b_{1}, \hat{\lambda}_{1} \in \mathbf{R}^{d}$, such that $\alpha \widehat{\lambda}_{1}$ is minimizer of $h_{C}$ and $h_{C}\left(\alpha \widehat{\lambda}_{1}\right)=-(1 / 2) \alpha^{2}\left\|b_{1}\right\|^{2}$.

Now because

$$
\min _{u \in \mathbf{R}^{d}} h(u)=\min _{C \in \mathscr{D}}\left\{\min _{w \in \mathbf{R}_{+}^{d}} h_{C}(w)\right\} .
$$

Note $\mathscr{D}$ is a finite set, first part of lemma holds.

If $\alpha>0$, take $D=D_{1} C$ and $b=-\left(B_{1} C^{+}-B_{2} C^{-}\right)^{T}$. because $D$ is nonsingular, by Lemma 1 again, there exist vectors $b_{2}, \hat{\lambda}_{2} \in \mathbf{R}^{d}$, such that $\alpha \hat{\lambda}_{2}$ is minimizer of $h_{C}$ and $h_{C}\left(\alpha \widehat{\lambda}_{2}\right)=(-1 / 2) \alpha^{2}\left\|b_{2}\right\|^{2}$.

Because (19) is true for this case, we have the second part of lemma. 
Note. (1) The minimizer may be not unique. In lemma $\hat{\lambda}_{1}, \hat{\lambda}_{2}$ is dependent only on the sign of $\alpha$.

(2) For $\alpha=0$, it is obvious that there is unique minimizer $u=0$ and $\min h(u)=0$.

Following the approach of LQ problem, we assume the value function is quadratic:

$$
V(t, x)=\frac{1}{2} P(t) x^{2}+g(t) x+c(t) .
$$

Note the coefficients $P(t), g(t), c(t)$ may be different for $\alpha$ positive and negative in Lemma 2.

Substituting (20) into HJB equation we have following ordinary differential equations (for $i=1,2$ ):

$$
\begin{gathered}
\dot{P}_{i}(t)=\left[-2 A(t)+\left\|b_{i}(t)\right\|^{2}\right] P_{i}(t), \\
P_{i}(T)=1, \\
\dot{g}_{i}(t)=\left[-A(t)+\left\|b_{i}(t)\right\|^{2}\right] g_{i}(t)-f(t) P_{i}(t), \\
g_{i}(T)=0, \\
\dot{c}_{i}(t)=-f(t) g_{i}(t)+\frac{1}{2}\left\|b_{i}(t)\right\|^{2} P_{i}(t)^{-1} g_{i}(t)^{2}, \\
c_{i}(T)=0 .
\end{gathered}
$$

Here $b_{1}(t), b_{2}(t)$ are determined by Lemmas 1 and 2 . If there are two solutions in some interval of $[0, T]$, we can always take same $C$ on that interval for it is piecewise constant. That makes our solution piecewise continuous.

Since $V_{x x}(t, x)=P_{i}(t)=e^{\int_{t}^{T}\left(2 A(s)-\left\|b_{i}(s)\right\|^{2}\right) d s}>0$, the $\alpha$ in Lemma 2 equals $V_{x}(t, x) / V_{x x}(t, x)$ having same sign as $V_{x}(t, x)$. By the lemmas, we know $b_{1}(t)$ and $b_{2}(t)$ are independent of $x, V_{x}(t, x)$ and $V_{x x}(t, x)$ except the sign of $V_{x}(t, x)$.

Defining $\eta_{i}(t):=g_{i}(t) / P_{i}(t), i=1,2$, from (21) and (22) we can get

$$
\begin{aligned}
\dot{\eta}_{i}(t) & =\frac{P_{i}(t) \dot{g}_{i}(t)-\dot{P}_{i}(t) g_{i}(t)}{P_{i}(t)^{2}} \\
& =\frac{A(t) P_{i}(t) g_{i}(t)-f(t) P_{i}(t)^{2}}{P_{i}(t)^{2}} \\
& =A(t) \eta_{i}(t)-f(t) .
\end{aligned}
$$

By the terminal constraint $\eta_{i}(T)=0$, noting the equations are same for $i=1,2$, we obtain

$$
\eta_{1}(t)=\eta_{2}(t)=e^{-\int_{t}^{T} A(s) d s} \int_{t}^{T} f(z) e^{\int_{z}^{T} A(s) d s} d z
$$

Therefore, for $i=1,2$,

$$
g_{i}(t)=P_{i}(t) \eta_{i}(t)=e^{\int_{t}^{T}\left(A(s)-\left\|b_{i}(s)\right\|^{2}\right) d s} \int_{t}^{T} f(z) e^{\int_{z}^{T} A(s) d s} d z
$$

Substituting these expressions into (23), we obtain for $i=1,2$

$$
\begin{aligned}
c_{i}(t)=\int_{t}^{T}\left\{\left[f(v)-\frac{1}{2}\left\|b_{i}(v)\right\|^{2} e^{-\int_{v}^{T} A(s) d s}\right.\right. \\
\left.\quad \times \int_{v}^{T} f(z) e^{\int_{z}^{T} A(s) d s} d z\right] \\
\left.\cdot e^{\int_{v}^{T}\left(A(s)-\left\|b_{i}(s)\right\|^{2}\right) d s} \int_{v}^{T} f(z) e^{\int_{z}^{T} A(s) d s} d z\right\} d v .
\end{aligned}
$$

Because

$$
\begin{aligned}
& \int_{t}^{T}\left\{f(v) e^{\int_{v}^{T}\left(A(s)-\left\|b_{i}(s)\right\|^{2}\right) d s} \int_{v}^{T} f(z) e^{\int_{z}^{T} A(s) d s} d z\right\} d v \\
& =\int_{t}^{T}\left\{e^{\int_{v}^{T}-\left\|b_{i}(s)\right\|^{2} d s}\left[f(v) e^{\int_{v}^{T} A(s) d s}\right]\right. \\
& \left.\times \int_{v}^{T} f(z) e^{\int_{z}^{T} A(s) d s} d z\right\} d v \\
& =-\frac{1}{2} \int_{t}^{T} e^{\int_{v}^{T}-\left\|b_{i}(s)\right\|^{2} d s} d\left[\int_{v}^{T} f(z) e^{\int_{z}^{T} A(s) d s} d z\right]^{2} \\
& =\frac{1}{2} e^{\int_{t}^{T}-\left\|b_{i}(s)\right\|^{2} d s}\left[\int_{t}^{T} f(z) e^{\int_{z}^{T} A(s) d s} d z\right]^{2} \\
& +\int_{t}^{T}\left\{\left\|b_{i}(v)\right\|^{2} \cdot e^{\int_{v}^{T}-\left\|b_{i}(s)\right\|^{2} d s}\right. \\
& \left.\times\left[\int_{v}^{T} f(z) e^{\int_{z}^{T} A(s) d s} d z\right]^{2}\right\} d v
\end{aligned}
$$

We have for $i=1,2$

$$
c_{i}(t)=\frac{1}{2} e^{\int_{t}^{T}-\left\|b_{i}(s)\right\|^{2} d s}\left[\int_{t}^{T} f(z) e^{\int_{z}^{T} A(s) d s} d z\right]^{2}
$$

3.2. Value Function and Optimal Control. Firstly, we will construct a viscosity solution for HJB equation. Let

$$
\eta(t):=e^{-\int_{t}^{T} A(s) d s} \int_{t}^{T} f(z) e^{\int_{z}^{T} A(s) d s} d z=\eta_{1}(t)=\eta_{2}(t) .
$$

Define the spaces

$$
\begin{aligned}
& \Gamma_{1}:=\{(t, x) \in[0, T] \times \mathbf{R} \mid x+\eta(t)<0\}, \\
& \Gamma_{2}:=\{(t, x) \in[0, T] \times \mathbf{R} \mid x+\eta(t)>0\}, \\
& \Gamma_{3}:=\{(t, x) \in[0, T] \times \mathbf{R} \mid x+\eta(t)=0\} .
\end{aligned}
$$

We denote $\alpha(t, x):=x+\eta(t)=x+\eta_{1}(t)=x+\eta_{2}(t)$. 
Now let us check that $V(t, x)=(1 / 2) P_{i}(t) x^{2}+g_{i}(t) x+c_{i}(t)$ satisfies HJB equation on $\Gamma_{i}$, for $i=1,2$. Considering (20) for $i=1$, we have

$$
\begin{gathered}
V_{t}(t, x)=\frac{1}{2} \dot{P}_{1}(t) x^{2}+\dot{g}_{1}(t) x+\dot{c}_{1}(t), \\
V_{x}(t, x)=P_{1}(t) x+g_{1}(t), \\
V_{x x}(t, x)=P_{1}(t) .
\end{gathered}
$$

Substitute into (11) for $(t, x) \in \Gamma_{1}$

LHS

$$
\begin{aligned}
& =V_{t}(t, x)+V_{x}(t, x)(A(t) x+f(t)) \\
& +\inf _{u \in \mathbf{R}^{d}}\left\{V_{x x}(t, x) \frac{1}{2} u^{T} D_{1}^{T} D_{1} u+V_{x}(t, x)\right. \\
& \left.\times\left(B_{1}(t) u^{+}-B_{2}(t) u^{-}\right)\right\} \\
& =\left[\frac{1}{2} \dot{P}_{1}(t) x^{2}+\dot{g}_{1}(t) x+\dot{c}_{1}(t)\right]+\left[P_{1}(t) x+g_{1}(t)\right] \\
& \times[A(t) x+f(t)]+\inf _{u \in \mathbb{R}^{d}}\left\{\frac{1}{2} P_{1}(t) u^{T} D_{1}^{T}(t) D_{1}(t) u\right. \\
& +\left[P_{1}(t) x+g_{1}(t)\right] \\
& \left.\times\left(B_{1}(t) u^{+}-B_{2}(t) u^{-}\right)\right\} \\
& =\left[\frac{1}{2} \dot{P}_{1}(t)+A(t) P_{1}(t)\right] x^{2} \\
& +\left[\dot{g}_{1}(t)+A(t) g_{1}(t)+f(t) P_{1}(t)\right] x \\
& +\left[\dot{c}_{1}(t)+f(t) g_{1}(t)\right]+P_{1}(t) \\
& \times \inf _{u \in \mathbf{R}^{d}}\left\{\frac{1}{2} u^{T} D_{1}^{T}(t) D_{1}(t) u+\left[x+\eta_{1}(t)\right]\right. \\
& \left.\times\left(B_{1}(t) u^{+}-B_{2}(t) u^{-}\right)\right\} \\
& =\left[\frac{1}{2} \dot{P}_{1}(t)+A(t) P_{1}(t)\right] x^{2} \\
& +\left[\dot{g}_{1}(t)+A(t) g_{1}(t)+f(t) P_{1}(t)\right] x \\
& +\left[\dot{c}_{1}(t)+f(t) g_{1}(t)\right]-P_{1}(t) \frac{1}{2}\left[x+\eta_{1}(t)\right]^{2} \\
& \times\left\|b_{1}(t, x)\right\|^{2} \\
& =\frac{1}{2}\left[\dot{P}_{1}(t)+2 A(t) P_{1}(t)-\left\|b_{1}(t, x)\right\|^{2} P_{1}(t)\right] x^{2} \\
& +\left[\dot{g}_{1}(t)+A(t) g_{1}(t)+f(t) P_{1}(t)\right.
\end{aligned}
$$

$$
\begin{aligned}
& \left.-P_{1}(t) \eta_{1}(t)\left\|b_{1}(t, x)\right\|^{2}\right] x \\
& +\dot{c}_{1}(t)+f(t) g_{1}(t)-\frac{1}{2} P_{1}(t)\left[\eta_{1}(t)\right]^{2}\left\|b_{1}(t, x)\right\|^{2} \\
& =0 .
\end{aligned}
$$

Since $\alpha(t, x)=x+\eta_{1}(t)<0$ in $\Gamma_{1}$, substituting the minimizer by Lemma 2 we can see $V(t, x)$ satisfies HJB equation in $\Gamma_{1}$.

By the same way, we can show $V(t, x)$ also satisfies HJB equation in $\Gamma_{2}$. For $i=2$, we have

$$
\begin{gathered}
V_{t}(t, x)=\frac{1}{2} \dot{P}_{2}(t) x^{2}+\dot{g}_{2}(t) x+\dot{c}_{2}(t), \\
V_{x}(t, x)=P_{2}(t) x+g_{2}(t), \\
V_{x x}(t, x)=P_{2}(t) .
\end{gathered}
$$

Substitute into (11) for $(t, x) \in \Gamma_{2}$

LHS

$$
\begin{aligned}
= & V_{t}(t, x)+V_{x}(t, x)(A(t) x+f(t)) \\
& +\inf _{u \in \mathbf{R}^{d}}\left\{V_{x x}(t, x) \frac{1}{2} u^{T} D_{1}^{T} D_{1} u+V_{x}(t, x)\right. \\
& \left.\quad \times\left(B_{1}(t) u^{+}-B_{2}(t) u^{-}\right)\right\} \\
= & {\left[\frac{1}{2} \dot{P}_{2}(t) x^{2}+\dot{g}_{2}(t) x+\dot{c}_{2}(t)\right] } \\
& +\left[P_{2}(t) x+g_{2}(t)\right][A(t) x+f(t)] \\
& +\inf _{u \in \mathbf{R}^{d}}\left\{\frac{1}{2} P_{2}(t) u^{T} D_{1}^{T}(t) D_{1}(t) u+\left[P_{2}(t) x+g_{2}(t)\right]\right. \\
& \left.\quad \times\left(B_{1}(t) u^{+}-B_{2}(t) u^{-}\right)\right\} \\
& +\left[\frac{1}{2}\left[x+\eta_{2}(t)\right]^{2}\left\|b_{2}(t, x)\right\|^{2}\right. \\
& +\left[\frac{1}{2} \dot{P}_{2}(t)+A(t) P_{2}(t)\right] x^{2}+\left[\dot{g}_{2}(t)+A(t) g_{2}(t)\right. \\
& +\left[\dot{c}_{2}(t)+f(t) g_{2}(t)\right]+P_{2}(t) \\
& \times \inf _{u \in \mathbf{R}^{d}}\left\{\frac{1}{2} u^{T} D_{1}^{T}(t) D_{1}(t) u+\left[x+\eta_{2}(t)\right]\right. \\
& \left.\quad f(t) P_{2}(t)\right] x
\end{aligned}
$$




$$
\begin{aligned}
= & \frac{1}{2}\left[\dot{P}_{2}(t)+2 A(t) P_{2}(t)-\left\|b_{2}(t, x)\right\|^{2} P_{2}(t)\right] x^{2} \\
+ & {\left[\dot{g}_{2}(t)+A(t) g_{2}(t)+f(t) P_{2}(t)\right.} \\
& \left.\quad-P_{2}(t) \eta_{2}(t)\left\|b_{2}(t, x)\right\|^{2}\right] x \\
& +\dot{c}_{2}(t)+f(t) g_{2}(t)-\frac{1}{2} P_{2}(t)\left[\eta_{2}(t)\right]^{2}\left\|b_{2}(t, x)\right\|^{2} \\
= & 0 .
\end{aligned}
$$

Since $x+\eta_{2}(t)>0$, substituting the minimizer we can see $V(t, x)$ satisfies HJB equation in $\Gamma_{2}$.

On $\Gamma_{3}$, we have $x=-\eta(t), g_{i}(t)=P_{i}(t) \eta(t)=-x P_{i}(t)$, then we have

$$
\begin{aligned}
V(t, x) & =\frac{1}{2} P_{i}(t) x^{2}+g_{i}(t) x+c_{i}(t) \\
& =\frac{1}{2} P_{i}(t) x^{2}-P_{i}(t) x^{2}+c_{i}(t)=0
\end{aligned}
$$

by (29), for $i=1,2$. So $V(t, x)$ is continuous at $(t, x) \in \Gamma_{3}$.

Therefore,

$$
\begin{gathered}
V_{t}(t, x)=\frac{1}{2} \dot{P}_{1}(t) x^{2}+\dot{g}_{1}(t) x+\dot{c}_{1}(t) \\
=\frac{1}{2} \dot{P}_{2}(t) x^{2}+\dot{g}_{2}(t) x+\dot{c}_{2}(t)=0, \\
V_{x}(t, x)=P_{1}(t) x+g_{1}(t)=P_{2}(t) x+g_{2}(t)=0,
\end{gathered}
$$

that is to say, $V(t, x)$ is differentiable on $\Gamma_{3}$. However, $V$ may be not smooth on $\Gamma_{3}$. It is clear $V_{x x}$ does not exist on $\Gamma_{3}$, since $P_{1}(t) \neq P_{2}(t)$. This means that the classical solution of HJB equation (11) does not exist. Therefore, we have to work within the framework of viscosity solution (see the appendix of [6]).

By the definition of viscosity solutions, we have for all $(t, x) \in \Gamma_{3}$,

$$
\begin{aligned}
& D_{t, x}^{1,2,+} V(t, x)=\{0\} \times\{0\} \times\left[P_{2}(t),+\infty\right), \\
& D_{t, x}^{1,2,-} V(t, x)=\{0\} \times\{0\} \times\left(-\infty, P_{1}(t)\right) .
\end{aligned}
$$

Now let

$$
G(t, x, u, p, P)=\frac{1}{2} P u^{T} D_{1}^{T} D_{1} u+p\left(B_{1} u^{+}-B_{2} u^{-}\right) .
$$

For all $(q, p, P) \in D_{t, x}^{1,2,+} V(t, x),(t, x) \in \Gamma_{3}$, we have

$$
\begin{aligned}
q & +\inf _{u \in \mathbf{R}^{d}} G(t, x, u, p, P) \\
& =\inf _{u \in \mathbf{R}^{d}}\left\{\frac{1}{2} P(t) u^{T} D_{1}^{T}(t) D_{1}(t) u\right\} \\
& \geq \inf _{u \in \mathbf{R}^{d}}\left\{\frac{1}{2} P_{2}(t) u^{T} D(t)^{T} D(t) u\right\}=0 .
\end{aligned}
$$

Therefore, $V$ is the subsolution of HJB equation (11). On the other hand, for all $(q, p, P) \in D_{t, x}^{1,2,-} V(t, x),(t, x) \in \Gamma_{3}$, we have

$$
\begin{aligned}
q & +\inf _{u \in \mathbf{R}^{d}} G(t, x, u, p, P) \\
& =\inf _{u \in \mathbf{R}^{d}}\left\{\frac{1}{2} P(t) u^{T} D_{1}^{T}(t) D_{1}(t) u\right\} \\
& \leq \inf _{u \in \mathbf{R}^{d}}\left\{\frac{1}{2} P_{1}(t) u^{T} D(t)^{T} D(t) u\right\}=0 .
\end{aligned}
$$

Therefore, by the definition we know $V(t, x)$ is the supersolution of HJB equation (11). Finally, it is easy to see that the terminal condition $V(T, x)=(1 / 2) x^{2}$ is satisfied. Therefore, by definition, $V(t, x)$ is the viscosity solution of HJB equation (11). Moreover, for all $(t, x) \in \Gamma_{3}$, let $\left(q^{*}(t, x)\right.$, $\left.p^{*}(t, x), P^{*}(t, x), u^{*}(t, x)\right):=\left(0,0, P_{2}(t), 0\right) \in D_{t, x}^{1,2,+} V(t, x) \times$ $u_{s}$ then

$$
q^{*}(t, x)+G\left(t, x, l^{*}(t, x), p^{*}(t, x), P^{*}(t, x)\right)=0
$$

So it satisfies the conditions in verification theorem [12, Theorem 3.1].

The main theorem in this section follows.

Theorem 3. Let $P_{i}(t), g_{i}(t), c_{i}(t)$ for $i=1,2$ be the solution of (21)-(23). The regions $\Gamma_{i}, i=1,2,3$ are defined by (31). Then the function defined by

$$
V(t, x)= \begin{cases}\frac{1}{2} P_{1}(t) x^{2}+g_{1}(t) x+c_{1}(t), & (t, x) \in \Gamma_{1}, \\ \frac{1}{2} P_{2}(t) x^{2}+g_{2}(t) x+c_{2}(t), & (t, x) \in \Gamma_{2} \cup \Gamma_{3}\end{cases}
$$

is the viscosity solution of HJB equation (11). Control function

$$
\pi^{*}(t, x)= \begin{cases}-\alpha(t, x) \hat{\lambda}_{1}(t), & (t, x) \in \Gamma_{1}, \\ -\alpha(t, x) \hat{\lambda}_{2}(t), & (t, x) \in \Gamma_{2} \cup \Gamma_{3}\end{cases}
$$

is the optimal feedback control of system (8) for objective functional (9) with $s=0$, the optimal value is $V(0, x)$. Here $\widehat{\lambda}_{1}, \widehat{\lambda}_{2}$ are constructed by Lemmas 1 and 2. 


\section{Optimal Strategy and Efficient Frontier}

4.1. Optimal Strategy. Back to the portfolio problem. From [6] problem (7) is equivalent to $A(\rho)$ for some $\rho$ :

$$
\begin{aligned}
& \min E\left\{\frac{1}{2}[X(T)-(\mu-\rho)]^{2}\right\} \\
& \text { s.t. }\left\{\begin{array}{l}
\pi(\cdot) \in L_{\mathscr{F}}^{2}\left(0, T, \mathbf{R}^{d}\right), \\
X(\cdot) \quad \text { satisfy }(5) .
\end{array}\right.
\end{aligned}
$$

In order to make use of the result in Section 3, let $x(t)=$ $X(t)-(\mu-\rho)$. We study optimal control problem

$$
\begin{aligned}
& \min \frac{1}{2} E\left\{x^{2}(T)\right\} \\
& \text { s.t. }\left\{\begin{aligned}
d x(t)= & {\left[r(t) x(t)+(b(t)-r(t) \mathbf{1})^{T} \pi(t)-\lambda r(t)\right.} \\
& \left.\times \mathbf{1}^{T} \pi^{-}(t)+(\mu-\rho) r(t)\right] d t \\
& +\pi^{T}(t) \sigma(t) d W(t), \\
x(0)= & v-(\mu-\rho) .
\end{aligned}\right.
\end{aligned}
$$

If we denote $A(t)=r(t), B_{1}(t)=b(t)-r(t) \mathbf{1}^{T}, B_{2}(t)=$ $\lambda r(t) \mathbf{1}^{T}, f(t)=(\mu-\rho) r(t), D_{1}(t)=\sigma^{T}(t),(46)$ becomes

$$
\begin{array}{ll}
\min \quad & \frac{1}{2} E\left\{x^{2}(T)\right\} \\
\text { s.t. } & \left\{\begin{array}{r}
d x(t)=\left[A(t) x(t)+B_{1}(t) \pi(t)-B_{0}(t) \pi^{-}(t)\right. \\
+f(t)] d t+\pi^{T}(t) D_{1}^{T}(t) d W(t), \\
x(0)=v-(\mu-\rho) .
\end{array}\right.
\end{array}
$$

Before analyzing the efficient frontier of the original problem (7), we firstly give the optimal strategy of $A(\rho)$. The translated problem is a special case studied in the previous section, the optimal strategy is given in (44). In this problem,

$$
\begin{aligned}
& \alpha(t, x) \\
& \quad=x+\eta(t)=x+e^{-\int_{t}^{T} r(s) d s} \int_{t}^{T}(\mu-\rho) d\left(-e^{\int_{z}^{T} r(s)}\right) d z \\
& \quad=x-e^{-\int_{t}^{T} r(s) d s}(\mu-\rho)\left(1-e^{\int_{t}^{T} r(s) d s}\right) \\
& \quad=x+(\mu-\rho)\left(1-e^{-\int_{t}^{T} r(s) d s}\right) .
\end{aligned}
$$

The optimal strategy is

$$
\pi^{*}(t, x)=\left\{\begin{array}{c}
-\hat{\lambda}_{1}(t)\left[x+(\mu-\rho)\left(1-e^{-\int_{t}^{T} r(s) d s}\right)\right], \\
\text { if } x+(\mu-\rho)\left(1-e^{-\int_{t}^{T} r(s) d s}\right)<0, \\
-\hat{\lambda}_{2}(t)\left[x+(\mu-\rho)\left(1-e^{-\int_{t}^{T} r(s) d s}\right)\right], \\
\text { if } x+(\mu-\rho)\left(1-e^{-\int_{t}^{T} r(s) d s}\right) \geq 0 .
\end{array}\right.
$$

Substituting $x$ with $X-(\mu-\rho)$, we get the following.
Theorem 4. For problem $A(\rho)$, the optimal portfolios are

$$
\pi^{*}(t, X)=\left\{\begin{array}{c}
-\widehat{\lambda}_{1}(t)\left[X-(\mu-\rho) e^{-\int_{t}^{T} r(s) d s}\right], \\
\text { if } X-(\mu-\rho) e^{-\int_{t}^{T} r(s) d s}<0, \\
-\widehat{\lambda}_{2}(t)\left[X-(\mu-\rho) e^{-\int_{t}^{T} r(s) d s}\right], \\
\text { if } X-(\mu-\rho) e^{-\int_{t}^{T} r(s) d s} \geq 0 .
\end{array}\right.
$$

Here $\widehat{\lambda}_{1}, \widehat{\lambda}_{2}$ are constructed by Lemmas 1 and 2 .

4.2. Efficient Frontier. In this part, we will compute the efficient frontier of problem $A(\rho)$. Because

$$
\begin{aligned}
\frac{1}{2} E & {\left[x(T)^{2}\right] } \\
& =\frac{1}{2} E[X(T)-(\mu-\rho)]^{2} \\
& =\frac{1}{2} E[X(T)-\mu]^{2}+\rho E[X(T)-\mu]+\frac{1}{2} \rho^{2} .
\end{aligned}
$$

For fixed $\rho$, we have

$$
\begin{aligned}
\min _{u(\cdot) \in u} E & \left\{\frac{1}{2}[X(T)-\mu]^{2}+\rho[E X(T)-\mu]\right\} \\
= & V(0, x(0))-\frac{1}{2} \rho^{2} \\
= & \frac{1}{2} P(0) x(0)^{2}+g(0) x(0)+c(0)-\frac{1}{2} \rho^{2} \\
= & \frac{1}{2} P(0)[v-(\mu-\rho)]^{2}+g(0)[v-(\mu-\rho)] \\
& +c(0)-\frac{1}{2} \rho^{2},
\end{aligned}
$$

where $P(\cdot), g(\cdot), c(\cdot)$ are either $P_{1}(\cdot), g_{1}(\cdot), c_{1}(\cdot)$ or $P_{2}(\cdot), g_{2}(\cdot)$, $c_{2}(\cdot)$, respectively, on $X-(\mu-\rho) e^{-\int_{t}^{T} r(s) d s}<0$ and $X-$ $(\mu-\rho) e^{-\int_{t}^{T} r(s) d s} \geq 0$. Now, if $X-(\mu-\rho) e^{-\int_{t}^{T} r(s) d s}<0$, we have a concave quadratic function in $\rho$

$$
\begin{aligned}
\min _{u(\cdot) \in u[0, T]} E\left\{\frac{1}{2}[X(T)-\mu]^{2}+\rho[E X(T)-\mu]\right\} \\
=\frac{1}{2} P_{1}(0)\left[X_{0}-(\mu-\rho)\right]^{2}+g_{1}(0)\left[X_{0}-(\mu-\rho)\right] \\
\quad+c_{1}(0)-\frac{1}{2} \rho^{2} \\
=\frac{1}{2} e^{-\int_{0}^{T}\left\|b_{1}(s)\right\|^{2} d s}\left[v e^{T_{0}^{T} r(s) d s}-(\mu-\rho)\right]^{2}-\frac{1}{2} \rho^{2} .
\end{aligned}
$$




$$
\begin{aligned}
& \text { If } X-(d-\rho) e^{-\int_{t}^{T} r(s) d s} \geq 0 \text {, we obtain } \\
& \begin{aligned}
\min _{u(\cdot) \in u[0, T]} E\left\{\frac{1}{2}[X(T)-\mu]^{2}+\rho[E X(T)-\mu]\right\} \\
=\frac{1}{2} P_{2}(0)\left[X_{0}-(\mu-\rho)\right]^{2}+g_{2}(0)\left[X_{0}-(\mu-\rho)\right] \\
\quad+c_{2}(0)-\frac{1}{2} \rho^{2} \\
=\frac{1}{2} e^{-\int_{0}^{T}\left\|b_{2}(s)\right\|^{2} d s}\left[v e^{\int_{0}^{T} r(s) d s}-(\mu-\rho)\right]^{2}-\frac{1}{2} \rho^{2} .
\end{aligned}
\end{aligned}
$$

The minimum variance (optimal value functional) is

$$
\begin{aligned}
& \min _{u(\cdot) \in u} E[X(T)-d]^{2}+2 \rho E[X(T)-d] \\
& =\left\{\begin{array}{c}
e^{-\int_{0}^{T}\left\|b_{1}(s)\right\|^{2} d s}\left[v e^{\int_{0}^{T} r(s) d s}-(\mu-\rho)\right]^{2}-\rho^{2}, \\
\text { if } v-(\mu-\rho) e^{-\int_{0}^{T} r(s) d s}<0 ; \\
e^{-\int_{0}^{T}\left\|b_{2}(s)\right\|^{2} d s}\left[v e^{\int_{0}^{T} r(s) d s}-(\mu-\rho)\right]^{2}-\rho^{2}, \\
\text { if } v-(\mu-\rho) e^{-\int_{t}^{T} r(s) d s} \geq 0 .
\end{array}\right.
\end{aligned}
$$

Since $\rho$ is Lagrange multiplier. We must maximize it. Note that $\mu \geq \mu_{0}=v e^{\int_{t}^{T} r(s) d s}$. If $v-(\mu-\rho) e^{-\int_{0}^{T} r(s) d s} \leq 0$, then $\rho \leq \mu-\mu_{0}$. Maximize

$$
e^{-\int_{0}^{T}\left\|b_{1}(s)\right\|^{2} d s}\left[v e^{\int_{0}^{T} r(s) d s}-(\mu-\rho)\right]^{2}-\rho^{2},
$$

we get

$$
\rho_{1}^{*}=\frac{\mu-\mu_{0}}{1-e^{\int_{0}^{T}\left\|b_{1}(s)\right\|^{2} d s}}<\mu-\mu_{0}
$$

with maximum value

$$
\frac{\left(\mu-\mu_{0}\right)^{2}}{e^{\int_{0}^{T}\left\|b_{1}(s)\right\|^{2} d s}-1} .
$$

If $v-(\mu-\rho) e^{-\int_{0}^{T} r(s) d s} \geq 0$, then $\rho \geq \mu-\mu_{0}$. Maximize

$$
e^{-\int_{0}^{T}\left\|b_{2}(s)\right\|^{2} d s}\left[v e^{\int_{0}^{T} r(s) d s}-(\mu-\rho)\right]^{2}-\rho^{2} .
$$

We get $\rho_{2}^{*}=\mu-\mu_{0}$ with maximum value $-\left(\rho_{2}^{*}\right)^{2}<0$. Therefore the maximum of (55) equals (58).

Now we have the theorem to conclude the section.

Theorem 5. The efficient strategy of mean-variance problem can be formulated as

$$
\pi^{*}(t, x)=\left\{\begin{array}{c}
-\hat{\lambda}_{1}(t)\left[x-\left(\mu-\rho^{*}\right) e^{\left.-\int_{t}^{T} r(s) d s\right],}\right. \\
\text { if } x-\left(\mu-\rho^{*}\right) e^{-\int_{t}^{T} r(s) d s}<0, \\
-\widehat{\lambda}_{2}(t)\left[x-\left(\mu-\rho^{*}\right) e^{\left.-\int_{t}^{T} r(s) d s\right],}\right. \\
\text { if } x-\left(\mu-\rho^{*}\right) e^{-\int_{t}^{T} r(s) d s} \geq 0,
\end{array}\right.
$$

where $\rho^{*}=\rho_{1}^{*}$ is defined in (53), $\hat{\lambda}_{1}, \hat{\lambda}_{2}$, come from Lemmas 1 and 2 . The minimum variance is

$$
\operatorname{var}[X(T)]=\frac{\left(\mu-\mu_{0}\right)^{2}}{e^{\int_{0}^{T}\left\|b_{1}(s)\right\|^{2} d s}-1} .
$$

Here $b_{1}$ is determined by Lemmas 1 and 2.

Note. When coefficients are all constant then $\widehat{\lambda}_{1}, \widehat{\lambda}_{2}$ and $b_{1}$ are all constant vectors.

\section{Two Examples}

Example 6. Suppose $d=1, T=1, r(t)=r, b(t)=b, \sigma(t)=1$. Then the wealth equation is

$$
\begin{gathered}
d X(t)=\left[r X(t)+(b-r) \pi(t)-\lambda r \pi^{-}(t)\right] d t+\pi(t) d W(t), \\
X(0)=v .
\end{gathered}
$$

The problem $A(\rho)$ can be formulated as

$$
\begin{array}{ll}
\min \quad \frac{1}{2} E\left\{x^{2}(T)\right\} \\
\text { s.t. }\left\{\begin{array}{c}
d x(t)=\left[\begin{array}{c}
r x(t)+(b-r) \pi(t)-\lambda r \pi^{-}(t) \\
+(\mu-\rho) r] d t+\pi(t) d W(t), \\
x(0)=v-(\mu-\rho) .
\end{array}\right.
\end{array}\right.
\end{array}
$$

The correspondence HJB equation is

$$
\begin{gathered}
v_{t}(t, x)+\inf _{u \in \mathbf{R}}\left\{v_{x}(t, x) g(t, x, u)+\frac{1}{2} v_{x x}(t, x) u^{2}\right\}=0, \\
v(T, x)=\frac{1}{2} x^{2}
\end{gathered}
$$

Here $g(t, x, u)=r x(t)+(b-r) u-\lambda r u^{-}+(\mu-\rho) r$. Letting $\alpha(t, x):=v_{x}(t, x) / v_{x x}(t, x)$, we need to minimize

$$
h(u)=\frac{1}{2} u^{2}+\left[(b-r) u-\lambda r u^{-}\right] .
$$

We assume $b>r>0$, then when $\alpha(t, x)<0$,

$$
\min h(u)=h(\alpha(t, x)(b-r))=-\frac{1}{2}[\alpha(t, x)(b-r)]^{2} .
$$

When $\alpha(t, x) \geq 0$,

$$
\begin{aligned}
\min h(u) & =h(-\alpha(t, x)(b-r+r \lambda)) \\
& =-\frac{1}{2}[\alpha(t, x)(b-r+r \lambda)]^{2} .
\end{aligned}
$$

By (53) we have the optimal $\rho^{*}=\left(\mu-\mu_{0}\right) /\left(1-e^{(b-r)^{2}}\right)$ (note $b_{1}^{2}=(b-r)^{2}$ and $\left.\mu_{0}=e^{r}\right)$ and the optimal value $(\mu-$ $\left.\mu_{0}\right)^{2} /\left(e^{(b-r)^{2}}-1\right)$; they are all independent of margin rate $\lambda$. 
Because $f(t)=\left(\mu-\rho^{*}\right) r$, we have $\Gamma_{3}=\{(t, x): x-(\mu-$ $\left.\left.\rho^{*}\right) e^{r(1-t)}=0\right\}$. By $(64)$ and $(65)$ we know $\widehat{\lambda}_{1}(t)=b-r, \widehat{\lambda}_{2}(t)=$ $-(b-r+\lambda r)$. By Theorem 5 , the efficient strategy of meanvariance problem can be formulated as

$$
\pi^{*}(t, x)=\left\{\begin{array}{c}
-(b-r)\left[x-\left(\mu-\rho^{*}\right) e^{-r(1-t)}\right], \\
\text { if } x-\left(\mu-\rho^{*}\right) e^{-r(1-t)}<0, \\
(b-r+\lambda r)\left[x-\left(\mu-\rho^{*}\right) e^{-r(1-t)}\right], \\
\text { if } x-\left(\mu-\rho^{*}\right) e^{-r(1-t)} \geq 0 .
\end{array}\right.
$$

It is interesting to see that although the optimal strategies are different when $\lambda$ changes, $\Gamma_{3}$ (which is the boundary of two regions $\Gamma_{1}$ and $\Gamma_{2}$ ) and the optimal values remain the same and therefore two regions $\Gamma_{1}, \Gamma_{2}$ and the efficient frontiers keep unchanged. But it is not always the same case, next example we will show that the optimal values and the efficient frontiers vary with margin rate $\lambda$.

Example 7. Let $d=2, T=1, r(t)=0.01, b(t)=\left(\begin{array}{ll}0.05 & 0.02\end{array}\right)$, $\sigma(t)=\left(\begin{array}{ll}2 & 0 \\ 1 & 2\end{array}\right)$. In order to solve the HJB equation, we must minimize

$$
\begin{aligned}
h(u)= & \frac{1}{2} u^{T} \sigma \sigma^{T} u+\alpha\left[(b-r \mathbf{1}) u-r \lambda u^{-}\right] \\
= & \frac{1}{2}\left(4 u_{1}^{2}+4 u_{1} u_{2}+5 u_{2}^{2}\right) \\
& +\alpha\left(0.04 u_{1}-0.01 \lambda u_{1}^{-}+0.01 u_{2}-0.01 \lambda u_{2}^{-}\right) .
\end{aligned}
$$

When $\lambda=0$, we have $u^{*}=-\alpha\left(\begin{array}{c}0.01125 \\ -0.0025\end{array}\right),\left\|b_{1}\right\|^{2}=$ 0.000425 .

When $\lambda=+\infty$, short-selling is not allowed. We have

$$
u^{*}(t, x)= \begin{cases}-\alpha(t, x)\left(\begin{array}{c}
0.01 \\
0
\end{array}\right), & \text { if } \alpha(t, x)<0, \\
\left(\begin{array}{l}
0 \\
0
\end{array}\right), & \text { if } \alpha(t, x) \geq 0 .\end{cases}
$$

$\left\|b_{1}\right\|^{2}=0.0004$.

When $\lambda=0.2$, we consider four cases: $u_{1}, u_{2} \geq 0 ; u_{1}$, $u_{2} \leq 0 ; u_{1} \geq 0, u_{2} \leq 0 ; u_{1} \leq 0, u_{2} \geq 0$, then take the minimum. We have

$$
u^{*}(t, x)= \begin{cases}-\alpha(t, x)\left(\begin{array}{c}
0.011 \\
-0.002
\end{array}\right), & \text { if } \alpha(t, x)<0, \\
-\alpha(t, x)\left(\begin{array}{c}
0.011875 \\
0.00275
\end{array}\right), & \text { if } \alpha(t, x) \geq 0 .\end{cases}
$$

$\left\|b_{1}\right\|^{2}=0.000416$.

From the example we know $b_{1}$ depends on $\lambda$. By Theorem 5, we know the optimal parameter $\rho^{*}$, the boundary of two regions $\Gamma_{1}$ and $\Gamma_{2}$, the optimal value are determined by $b_{1}$ therefore they all vary with $\lambda$. For the same return $\mu$, the risk is lowest when $\lambda=0$ (i.e., no margin required) and the risk is highest when short-selling is prohibited $\lambda=+\infty$. The results of our paper cover these two extreme cases.

\section{Conclusion}

In this paper, we study the mean-variance portfolio selection problems for short selling with margin required. The problem is more complicated than free short-selling $(\lambda=0)$ and short-selling prohibiting $(\lambda=+\infty)$. We found the optimal strategies in the feedback form and the optimal value function which is the viscosity solution of the HJB equation. We have also given examples to show how the margin rate affects the optimal portfolio, optimal variance, and efficient frontier.

\section{Acknowledgment}

This work was supported by the National Natural Science Foundation of China (10971127).

\section{References}

[1] H. Markowitz, "Portfolio selection," Journal of Finance, vol. 7, no. 1, pp. 77-91, 1952.

[2] R. Merton, "An analytic derivation of the efficient portfolio frontier," Journal of Financial and Quantitative Analysis, vol. 7, pp. 1851-1872, 1972.

[3] D. Li and W.-L. Ng, "Optimal dynamic portfolio selection: multiperiod mean-variance formulation," Mathematical Finance, vol. 10, no. 3, pp. 387-406, 2000.

[4] D. Duffie and H. R. Richardson, "Mean-variance hedging in continuous time," The Annals of Applied Probability, vol. 1, no. 1, pp. 1-15, 1991.

[5] X. Y. Zhou and D. Li, "Continuous-time mean-variance portfolio selection: a stochastic LQ framework," Applied Mathematics and Optimization, vol. 42, no. 1, pp. 19-33, 2000.

[6] X. Li, X. Y. Zhou, and A. E. B. Lim, "Dynamic mean-variance portfolio selection with no-shorting constraints," SIAM Journal on Control and Optimization, vol. 40, no. 3, pp. 1540-1555, 2002.

[7] D. Cuoco and H. Liu, "A martingale characterization of consumption choices and hedging costs with margin requirements," Mathematical Finance, vol. 10, no. 3, pp. 355-385, 2000.

[8] D. C. Heath and R. A. Jarrow, "Arbitrage, continuous trading, and margin requirements," The Journal of Finance, vol. 42, no. 5, pp. 1129-1142, 1987.

[9] J. Liu and F. A. Longstaff, "Losing money on arbitrage: optimal dynamic portfolio choice in markets with arbitrage opportunities," Review of Financial Studies, vol. 17, no. 3, 2004.

[10] G.-L. Xu and S. E. Shreve, "A duality method for optimal consumption and investment under short-selling prohibition. II. Constant market coefficients," The Annals of Applied Probability, vol. 2, no. 2, pp. 314-328, 1992.

[11] J. Yong and X. Y. Zhou, Stochastic Controls: Hamlitonian Systems and HJB Equations, vol. 43, Springer, New York, NY, USA, 1999.

[12] X. Y. Zhou, J. Yong, and X. Li, "Stochastic verification theorems within the framework of viscosity solutions," SIAM Journal on Control and Optimization, vol. 35, no. 1, pp. 243-253, 1997. 


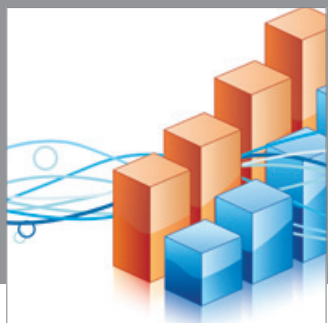

Advances in

Operations Research

mansans

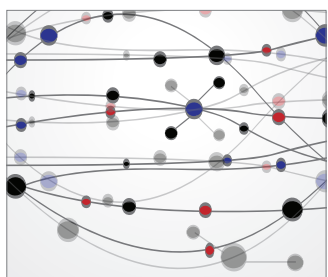

The Scientific World Journal
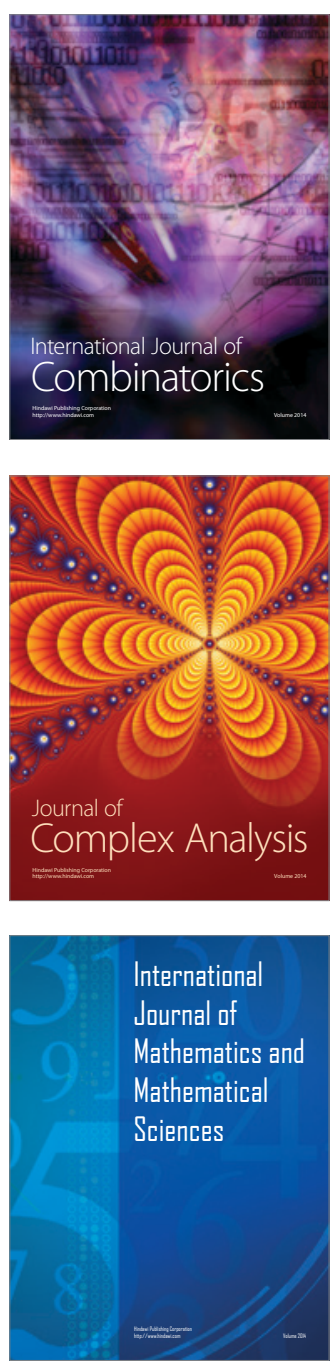
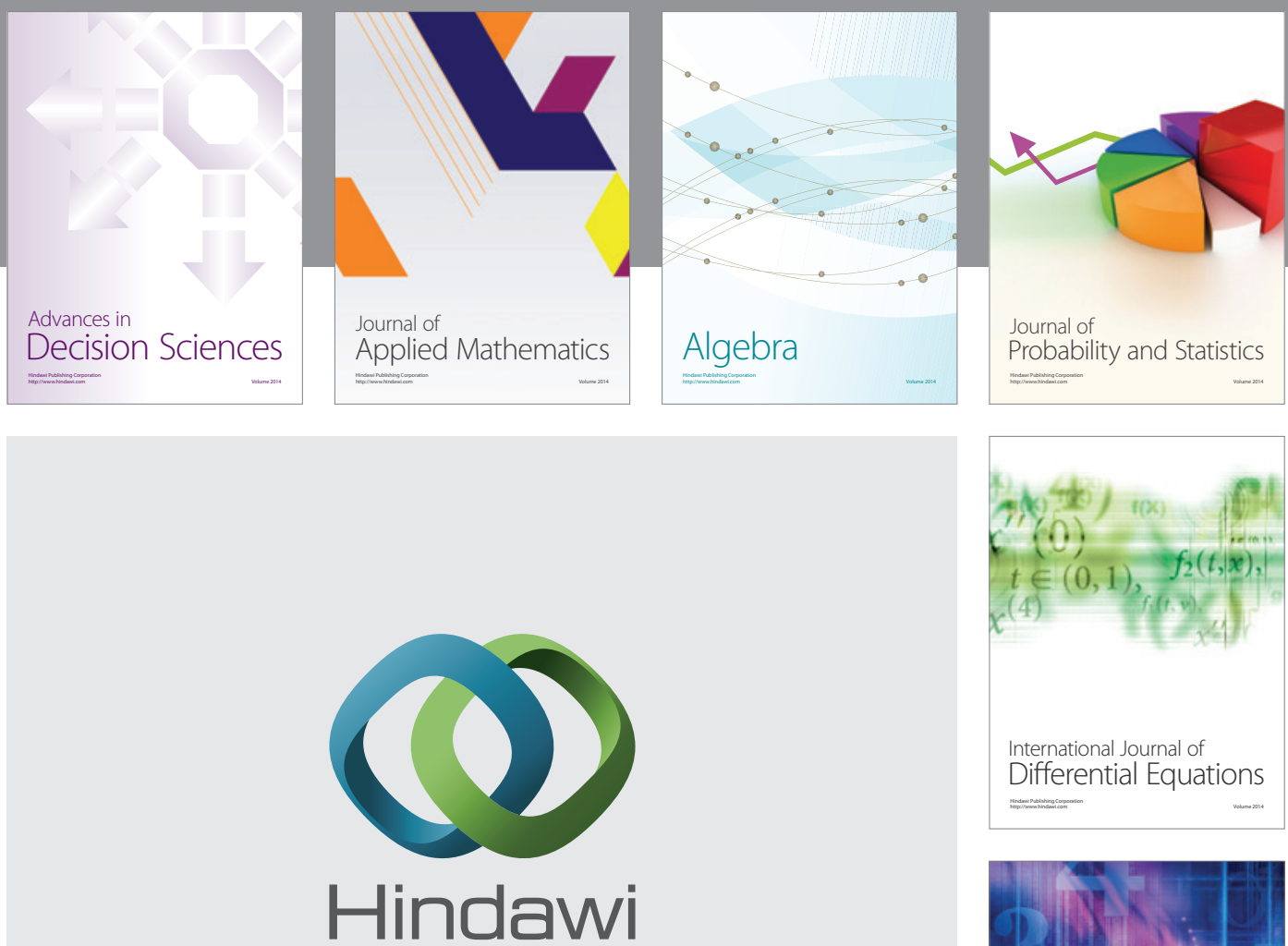

Submit your manuscripts at http://www.hindawi.com
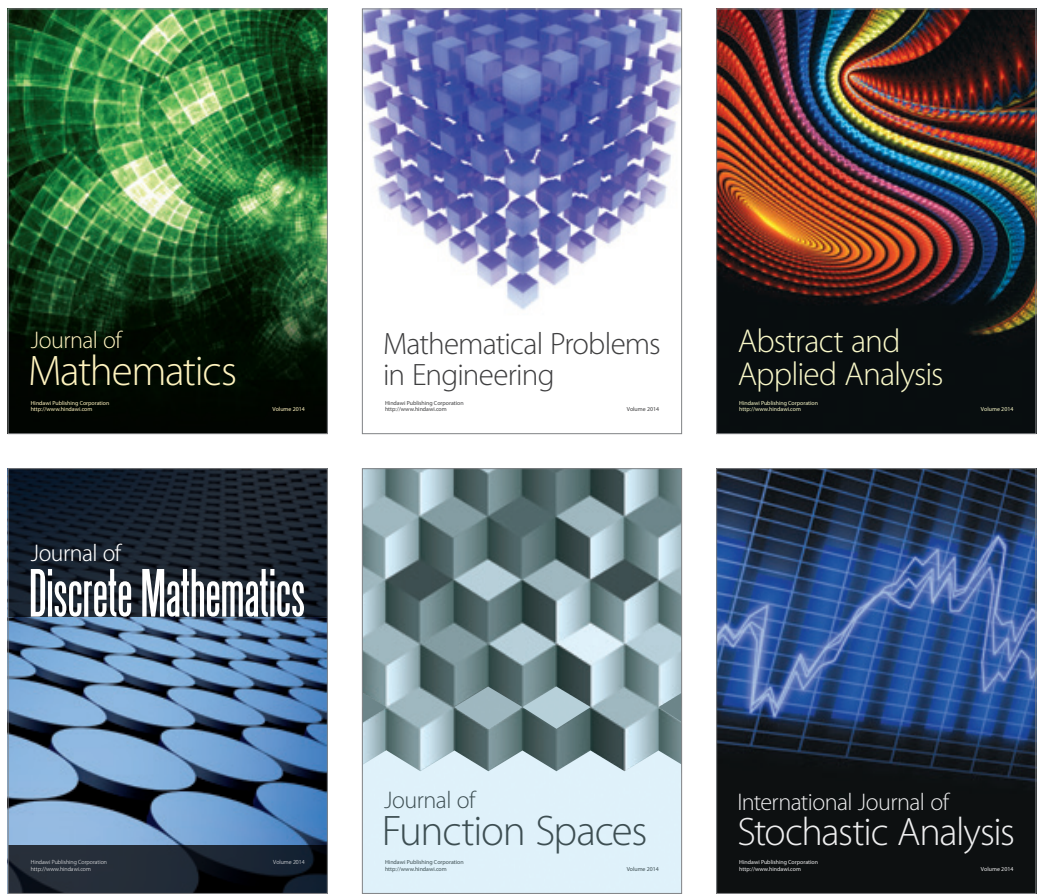

Journal of

Function Spaces

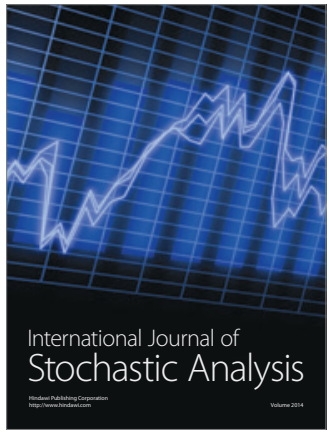

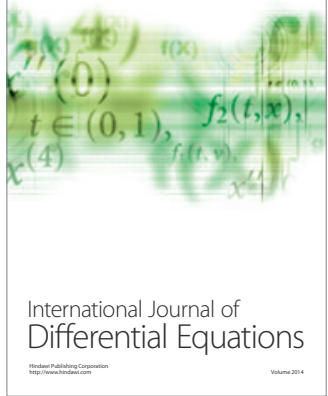
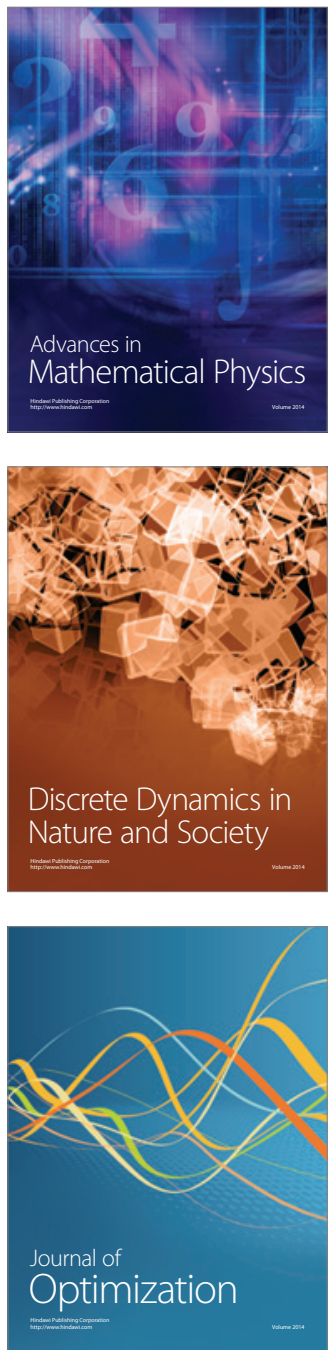